\title{
MicroRNA-3619-5p suppresses bladder carcinoma progression by directly targeting $\beta$-catenin and CDK2 and activating p21
}

\author{
Qingsong Zhang ${ }^{1,2}$, Shuo Miao ${ }^{3}$, Xihong $\mathrm{Han}^{4}$, Chuanchang Li ${ }^{1}$, Mengyang Zhang ${ }^{1}$, Kai Cui ${ }^{1}$, Tao Xiong ${ }^{1}$, \\ Zhong Chen', Chenghe Wang ${ }^{5}$ and Hua $\mathrm{Xu}^{1}$
}

\begin{abstract}
Current studies indicate that microRNAs (miRNAs) are widely decreased in various tumors and function as tumor suppressors by inhibiting cancer cell proliferation, survival, invasion, and migration. The potential application of using miRNAs to predict therapeutic responses to multiple types of cancer treatment holds high promise. In current study, we demonstrate that miR-3619-5p is downregulated in bladder cancer (BCa) tissues and cells. Exogenous overexpression of miR-3619-5p in BCa cells inhibits proliferation, migration, and invasion. Moreover, a nude mouse xenograft model shows that miR-3619-5p inhibits BCa cell growth. We also demonstrate that miR-3619-5p leads to the activation of p21 by targeting its promoter in BCa cells. Enforced miR-3619-5p expression consistently leads to the downregulation of $\beta$-catenin and cyclin-dependent kinase 2 (CDK2) through predicted binding sites within the $\beta$ catenin and CDK2 $3^{\prime}$-untranslated regions (UTRs), respectively. Moreover, $\beta$-catenin and CDK2 knockdown is able to mimic BCa cells growth and metastasis effects induced by overexpressing miR-3619-5p. We further confirm that miR3619-5p inhibits Wnt- $\beta$-catenin signal pathway and EMT progression in BCa cells. We also found that miR-3619-5pinduced growth arrest and metastasis inhibition are p21-dependent in BCa cells. Taken together, these results confirm that miR-3619-5p plays a tumor suppressive role in BCa by interfering with cell growth and metastasis and may serve as a potential therapeutic target in BCa treatment.
\end{abstract}

\section{Introduction}

Bladder cancer $(\mathrm{BCa})$ is one of the most common urological malignancy, and the incidence of $\mathrm{BCa}$ is expected to rise globally ${ }^{1}$. There are approximate 430,000 newly diagnosed cases every year all over the world and $\mathrm{BCa}$ is a common cause of cancer-related death among urinary tumors in China ${ }^{2}$. Although multiple treatments have

\footnotetext{
Correspondence: Zhong Chen (chenzhongtongji@126.com)

${ }^{1}$ Department of Urology, Tongji Hospital, Tongji Medical College, Huazhong University of Science and Technology, No. 1095 JieFang Avenue, 430030

Wuhan, Hubei, China

${ }^{2}$ Department of Urology, The Affiliated Hospital of Qingdao University, No. 16

Jiangsu Road, Shinan District, 26600 Qingdao, Shandong, China

Full list of author information is available at the end of the article.

Edited by A Peschiaroli.
}

been gained, the 5-year survival rate of $\mathrm{BCa}$ patients is still dissatisfied $^{3}$. About $33-75 \%$ of patients with $\mathrm{BC}$ a failed to respond to therapy due to the disease relapse or metastasis ${ }^{4}$. There is an urgent need for further investigation of the carcinogenesis and development of BCa. Regulation of specific tumor suppressor genes was confirmed to largely contribute to $\mathrm{BCa}$ initiation, proliferation, and metastasis; these results have led the scholars to research novel therapies based on targeted gene therapy for cancer treatment ${ }^{5}$.

miRNAs are a cluster of small endogenous noncoding RNAs composed of approximately 19-24 nucleotides that regulate target genes post-transcriptionally ${ }^{6}$. miRNAs play a key role in tumor cells growth, differentiation, 
metastasis, and apoptosis ${ }^{7,8}$. Increasing evidence has shown that miRNAs are involved in the progression of multiple types of cancers, including hepatocellular carcinoma, gastric cancer, glioma, and $\mathrm{BCa}^{9-12}$. In this regard, miRNAs are considered to be pivotal regulators of genes expression.

It is recently reported that the $\mathrm{Wnt} / \beta$-catenin signaling pathway is associated with $\mathrm{BCa}$ cell proliferation and differentiation ${ }^{13}$. Additionally, miRNAs are able to inhibit $\mathrm{BCa}$ cell epithelial-mesenchymal transition (EMT), which plays a crucial role in the early stages of proliferation and invasiveness ${ }^{14,15}$. In this study, we discovered that miR-3619 was decreased in both BCa cell lines and $\mathrm{BCa}$ clinical specimens. Enforced miR-3619 expression interfered with cell proliferation and metastasis and promoted cellular senescence and apoptosis; tumor growth in vivo was also suppressed. Furthermore, $\mathrm{BCa}$ cell proliferation and metastasis abilities were boosted by silencing endogenous miR-3619. Moreover, we demonstrated that CDK2 and $\beta$-catenin, both of which are direct miR-3619 target genes, played very important roles in BCa cell growth and metastasis. We also confirmed that miR-3619 activated p21 expression, which has a potent ability to suppress $\mathrm{BCa}$ progression ${ }^{16}$ by binding to its specific promoter. Together, our results provided new evidence that miR-3619 overexpression inhibited $\mathrm{BCa}$ progression and might represent a novel therapeutic target for $\mathrm{BCa}$ treatment.

\section{Results}

miR-3619 and p21 expression are reduced in both BCa tissues and BCa cell lines and associated with cancer progression

As shown in Fig. 1a, b, miR-3619 and p21 mRNA and protein levels were significantly downregulated in four BCa cell lines (5637, EJ, T24, and J82) compared with bladder mucosa epithelial cell line SV-HUC-1 cells. In $\mathrm{BCa}$ tissues, the mean score of $\mathrm{p} 21$ in tumor tissues was much lower than that in normal tissues, $2.806 \pm 0.3649$ vs. $5.812 \pm 0.6483(P<0.0001$, Wilcoxon signed rank test) (Fig. 1c). And the average expression levels of miR3619 and p21 mRNA levels were also lower in the BCa tissues than in the adjacent normal tissues (Fig. 1d). Spearman's correlation test was used to evaluate the relationship between miR-3619 and p21 expression levels. The outcomes showed that miR-3619 expression was positively correlated with p21 expression (Supplementary Figure 1A, Tumor, $r=0.64, P<0.0001$; Normal, $r=0.61, P=0.0016)$. Besides, miR-3619 and $\mathrm{p} 21$ expression $(\mathrm{T} / \mathrm{N})$ were correlated with tumor stage and grade $(P<0.05)$ and $\mathrm{BCa}$ clinicopathological factors were discussed and shown in Table 1 . These data indicated that miR-3619 and p21 might act as tumor suppressors in $\mathrm{BCa}$.
Low expression of p21 gene or decrease of both p21 and miR-3619 is associated with poor overall survival (OS) in BCa patients

We analyzed the relationship between p21 gene expression and $\mathrm{BCa}$ patients' OS. The median fold change $(\mathrm{T} / \mathrm{N})$ in $\mathrm{p} 21$ expression used as cutoff value; patients with high p21 expression had longer survival time than patients with low p21 expression (Fig. 1e, $\mathrm{HR}=5.36,95 \%$ CI 2.13-22.58; $P=0.027)^{17}$. Multivariate Cox analysis was performed to detect whether p21 gene was an independent prognostic factor in $\mathrm{BCa}$ patients. The outcomes showed that downregulated p21 was associated with poor $\mathrm{OS}$ in $\mathrm{BCa}(\mathrm{HR}=4.38,95 \% \mathrm{CI} 1.36-11.02 ; P=0.048)$ independent of other covariates (Table 2), which suggested that p21 gene in $\mathrm{BCa}$ patients might be an independent prognostic factor. Furthermore, the Kaplan - Meier curves demonstrated that high expression of p21 or miR-3619 in BCa patients survived longer than patients with low p21 and miR-3619 expression (Fig. 1f, $\mathrm{HR}=$ 5.68, 95\% CI 2.48-32.71; $P=0.013)$. Similarly, the multivariate Cox regression model displayed that there was a positive correlation between the low levels of miR-3619 and p21 expression and the poor OS in BCa patients (HR $=5.66$, 95\% CI 1.05-23.64; $P=0.036)$ independent of other clinicopathological factors (Table 2). Additionally, the univariate and multivariate Cox regression analysis results showed that tumor grades $(\mathrm{G} 2-3)$ and TNM stage $(\mathrm{T} 2-4)$ were associated with poor OS $(P<0.05)$ in the 33 BCa patients.

\section{$\beta$-catenin and CDK2 are overexpressed in human BCa tissues and cell lines and negatively correlated with miR- 3619 expression}

The expression of $\beta$-catenin and CDK2 proteins was remarkably increased in the four $\mathrm{BCa}$ cell lines compared with SV-HUC-1 cells (Fig. 1g). IHC and western blotting were performed to detect the protein expression characteristics of $\beta$-catenin and CDK2 in the $\mathrm{BCa}$ and para-carcinoma tissues. The western blotting results showed that $\beta$-catenin and CDK2 proteins had higher expression in the $\mathrm{BCa}$ tissues than in the adjacent normal tissues (Fig. 1h). The IHC results showed a significant enhancement of $\beta$-catenin and CDK2 expression in $\mathrm{BCa}$ tissues compared with adjacent normal tissues. The mean scores of $\beta$-catenin were elevated from $3.903 \pm 0.5147$ to $6.155 \pm 0.6587(P<0.01$, Wilcoxon signed rank test). Similarly, the mean scores of CDK2 in tumor tissues and adjacent normal tissues were $7.018 \pm 0.6516$ and $3.644 \pm 0.5090$, respectively $(P<$ 0.0001, Wilcoxon signed rank test) (Fig. 1i). We also observed that $\beta$-catenin and $\mathrm{CDK} 2$ expression were significantly overexpressed in the $\mathrm{BCa}$ samples in comparison with the normal bladder samples using Oncomine (www.oncomine.org) from original published data 


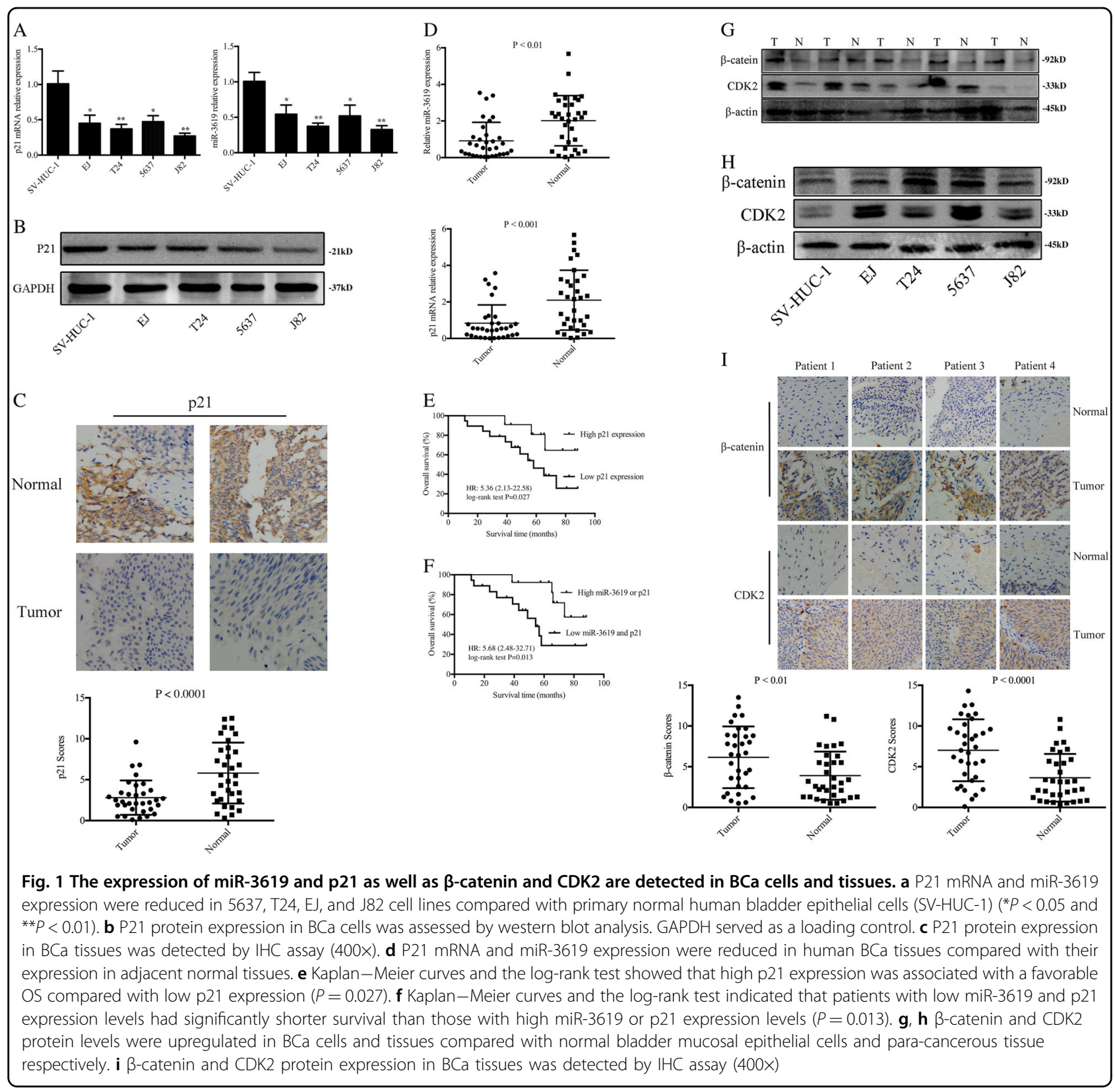

(Supplementary Figure 1B). We found a strong inverse correlation between miR-3619 and $\beta$-catenin and between miR-3619 and CDK2 (Supplementary Figure $1 C, r=-0.45, P=0.009 ; r=-0.47, P=0.0058$ ), indicating the presence of a regulatory relationship between miR-3619 and CDK2/ $\beta$-catenin in BCa cells.

\section{miR-3619 inhibits BCa cell proliferation and survival in vitro and in vivo}

As shown in Fig. 2a, compared to the dsControl group, both tested cells exhibited progressive retarded growth after $48 \mathrm{~h}$ as measured by CellTiter $96^{\circledR} \mathrm{AQ}_{\text {ueous }}$ One Solution Cell Proliferation Assay. An EdU assay was conducted to further detect the alterations in cell proliferation, and the outcomes were consistent with the above results (Fig. 2b, c). The 5637 and T24 cells both showed less colony formation after enforced miR-3619 expression (Fig. 2d). Besides, the 5637 and T24 cells accumulated in G1/G0 phase and displayed reduced S phase and G2/M phase cells after miR-3619 transfection (Fig. 2e). We found that upregulation of miR-3619 increased 5637 and T24 cells' apoptosis in both early and late stage as measured by flow cytometry with PI and Annexin V staining (Fig. 2f). Subsequently, we tested whether proliferation inhibition by miR-3619 was associated with senescence. We measured senescence- 
Table 1 Associations between expression of miR-3619, p21 and clinicopathologic factors in patients with BCa (n= 33)

\begin{tabular}{|c|c|c|c|c|c|}
\hline Parameter & Number of case & miR-3619 expression & $P$ value & p21expression & $P$ value \\
\hline \multicolumn{6}{|l|}{ Age, y } \\
\hline$>60$ & 19 & $0.51 \pm 0.31$ & 0.83 & $0.58 \pm 0.24$ & 0.54 \\
\hline$\leq 60$ & 14 & $0.49 \pm 0.26$ & & $0.66 \pm 0.29$ & \\
\hline \multicolumn{6}{|l|}{ Sex } \\
\hline Male & 20 & $0.42 \pm 0.24$ & 0.47 & $0.68 \pm 0.22$ & 0.96 \\
\hline Female & 13 & $0.47 \pm 0.27$ & & $0.70 \pm 0.17$ & \\
\hline \multicolumn{6}{|c|}{ Tumor diameter, cm } \\
\hline$\leq 3$ & 19 & $0.52 \pm 0.27$ & 0.13 & $0.69 \pm 0.25$ & 0.17 \\
\hline$>3$ & 14 & $0.43 \pm 0.22$ & & $0.56 \pm 0.33$ & \\
\hline \multicolumn{6}{|l|}{ Stage } \\
\hline $\mathrm{Ta}$ & 15 & $0.57 \pm 0.22$ & 0.024 & $0.72 \pm 0.25$ & 0.006 \\
\hline $\mathrm{T} 1$ & 13 & $0.44 \pm 0.17$ & & $0.55 \pm 0.15$ & \\
\hline $\mathrm{T} 2-4$ & 5 & $0.37 \pm 0.11$ & & $0.43 \pm 0.18$ & \\
\hline \multicolumn{6}{|l|}{ Grade } \\
\hline G1 & 23 & $0.54 \pm 0.28$ & 0.022 & $0.75 \pm 0.21$ & 0.009 \\
\hline G2/G3 & 10 & $0.35 \pm 0.16$ & & $0.47 \pm 0.28$ & \\
\hline
\end{tabular}

Table 2 Univariate and multivariate analysis of various prognostic variables and overall survival (OS) in patients with $\mathrm{BCa}(n=33)$

\begin{tabular}{|c|c|c|c|c|}
\hline \multirow[t]{2}{*}{ Variables (and stratification) } & \multicolumn{2}{|c|}{ Univariate analysis } & \multicolumn{2}{|c|}{ Multivariate analysis } \\
\hline & HR $(95 \% \mathrm{Cl})$ & $P$ value & HR $(95 \% \mathrm{CI})$ & $P$ value \\
\hline Age (>60 vs. $\leq 60$ y) & $1.42(0.64-2.38)$ & 0.55 & - & - \\
\hline Sex (male vs. female) & $1.11(0.55-3.61)$ & 0.68 & - & - \\
\hline BMI (>25.51 vs. $<25.51 \mathrm{~kg} / \mathrm{m}^{2}$ ) & $2.57(0.77-5.80)$ & 0.17 & - & - \\
\hline Stage (T2-4 vs. Ta-1) & $5.74(1.35-18.72)$ & 0.014 & $5.15(1.33-15.24)$ & 0.023 \\
\hline Grade (G2-3 vs. G1) & $6.31(2.21-24.58)$ & 0.0087 & $5.72(1.28-19.83)$ & 0.019 \\
\hline Tumor size (>3 vs. $\leq 3 \mathrm{~cm}$ ) & $4.14(1.27-15.90)$ & 0.074 & - & - \\
\hline miR-3619 expression (low vs. high) & $4.27(1.09-12.75)$ & 0.053 & - & - \\
\hline p21 expression (low vs. high) & $5.36(2.13-22.58)$ & 0.027 & $4.38(1.36-11.02)$ & 0.048 \\
\hline Low miR-3619 and p21 vs. high miR-3619 or p21 & $5.68(2.48-32.71)$ & 0.013 & $5.66(1.05-23.64)$ & 0.036 \\
\hline
\end{tabular}

associated $\beta$-galactosidase activity (SA- $\beta$-Gal) activity and found a marked increase in positive $\beta$-galactosidase cells following miR-3619 transfection (Fig. 2g). As shown in Fig. 2h, miR-3619 overexpression remarkably delayed T24 xenograft tumor growth. Besides, the reduction and loss in the average tumor volume and weight of miR-3619- overexpressing xenografts were compared with those of the control group.

miR-3619 restrain $\mathrm{BCa}$ cell migration and invasion

A wound-healing assay was performed to assess the migration capacity of 5637 and T24 cells transfected with 


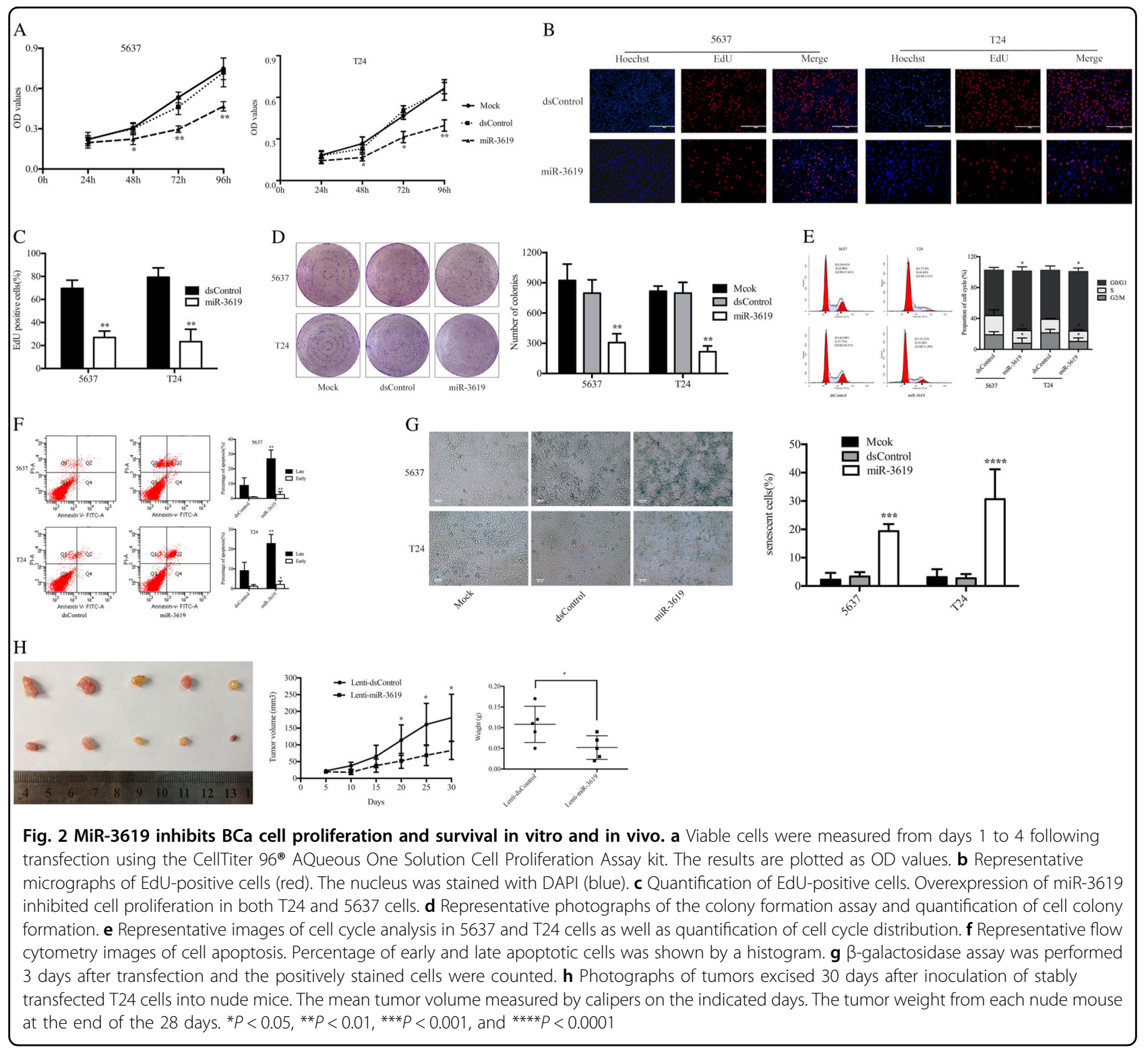

miR-3619 mimics. The results showed that miR-3619 upregulation inhibited cell migration (Fig. 3a). We also conducted migration and Matrigel invasion chamber assays to evaluate the migration and invasion abilities of the transfected cells, respectively. In accordance with the wound-healing assay results, $\mathrm{BCa}$ cells transfected with miR-3619 mimics showed suppressed migration and invasion compared with the mock or dsControl group (Fig. 3b, c).

\section{Enforcing miR-3619 expression overexpresses p21 and downregulates $\beta$-catenin and CDK2 while inhibiting epithelial-mesenchymal transition}

By querying the http://www.microrna.org ${ }^{18}$ and TargetScan $^{6}$ (http://www.targetscan.org) miRNA databases and searching for miRNA target screening using the miRanda algorithm ${ }^{19}$, we identified $\mathrm{p} 21, \beta$-catenin, and CDK2 as candidate miR-3619 targets. To confirm the influence of miR-3619 on p21, $\beta$-catenin and CDK2 in BCa cells, we transfected miR-3619 mimics into 5637 and T24 cells and analyzed p21, $\beta$-catenin and CDK2 expression after $72 \mathrm{~h}$. The transfection ratios were tested by qRT-PCR (Supplementary Figure 2A). The levels of p21, $\beta$-catenin, CDK2 and each downstream target were detected after reinforced miR-3619 expression. As shown in Fig. 4a, the mRNA levels revealed a profound p21 and E-cadherin induction and Cyclin D1 suppression after miR-3619 transfection. General PCR was performed and the results were similar to the qRT-PCR results (Supplementary Figure 2B). p21, E-cadherin, and Cyclin D1 


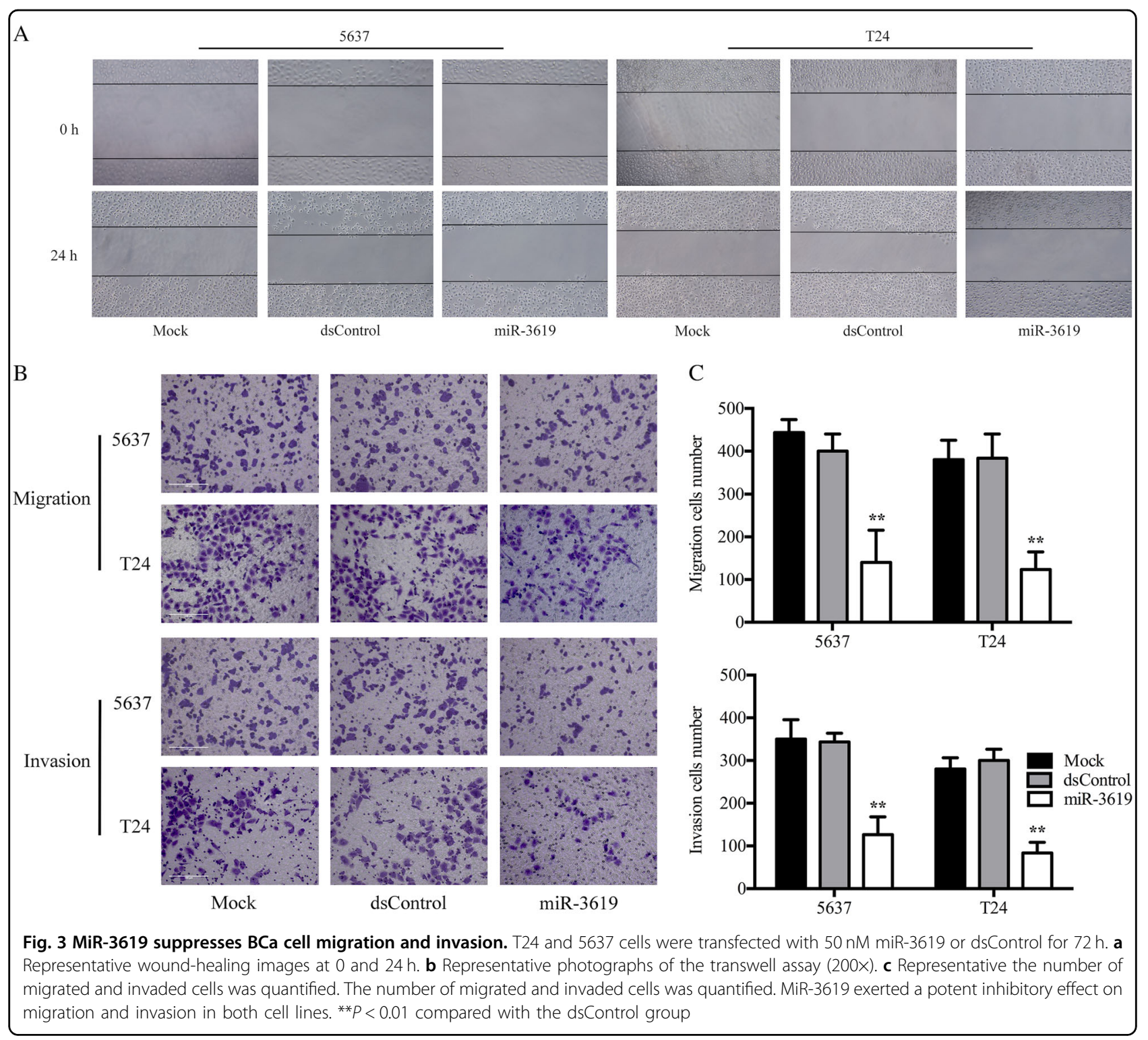

protein expression was further confirmed by immunoblot analysis (Fig. 4b). $\beta$-catenin and CDK2 protein expression were decreased and the expression of the downstream genes C-myc and MMP9 were also downregulated after miR-3619 transfection into the two cell lines (Fig. 4c).

Moreover, as $\beta$-catenin's nuclear translocation is a hallmark that activates $\mathrm{Wnt} / \beta$-catenin signaling ${ }^{20}$, immunofluorescence on 5637 and T24 cells was performed to further corroborate the nuclear localization of $\beta$-catenin. The results showed that nuclear $\beta$-catenin was decreased after miR-3619 overexpression (Fig. 4d, e). These data supported that $\mathrm{p} 21, \beta$-catenin, and CDK2 might be direct targets of miR-3619. As seen from Fig. 4a, f compared to the dsControl group, miR-3619 statistically increased the mRNA expression of the epithelial marker E-cadherin and also decreased the mesenchymal markers
$\mathrm{N}$-cadherin, Vimentin, and Snail in 5637 and T24 cells. General PCR was conducted and the outcomes were consistent with the qRT-PCR results (Supplementary Figure 2C). Western blotting also showed a robust upregulation in E-cadherin and downregulation in $\mathrm{N}$-cadherin, Vimentin, and Snail protein levels in both 5637 and T24 cells after miR-3619 transfection (Fig. 4b, g).

\section{MiR-3619 interacts with the p21 gene promoter and directly targets the predicted binding sites in the $\beta$-catenin and CDK2 $3^{\prime}$-UTRs}

Studies have verified that the binding of miRNAs to target gene promoters is a possible mechanism for RNA activation $^{21-23}$. ChIP assay was conducted to identify whether miR-3619 activated p21 by targeting a specific 


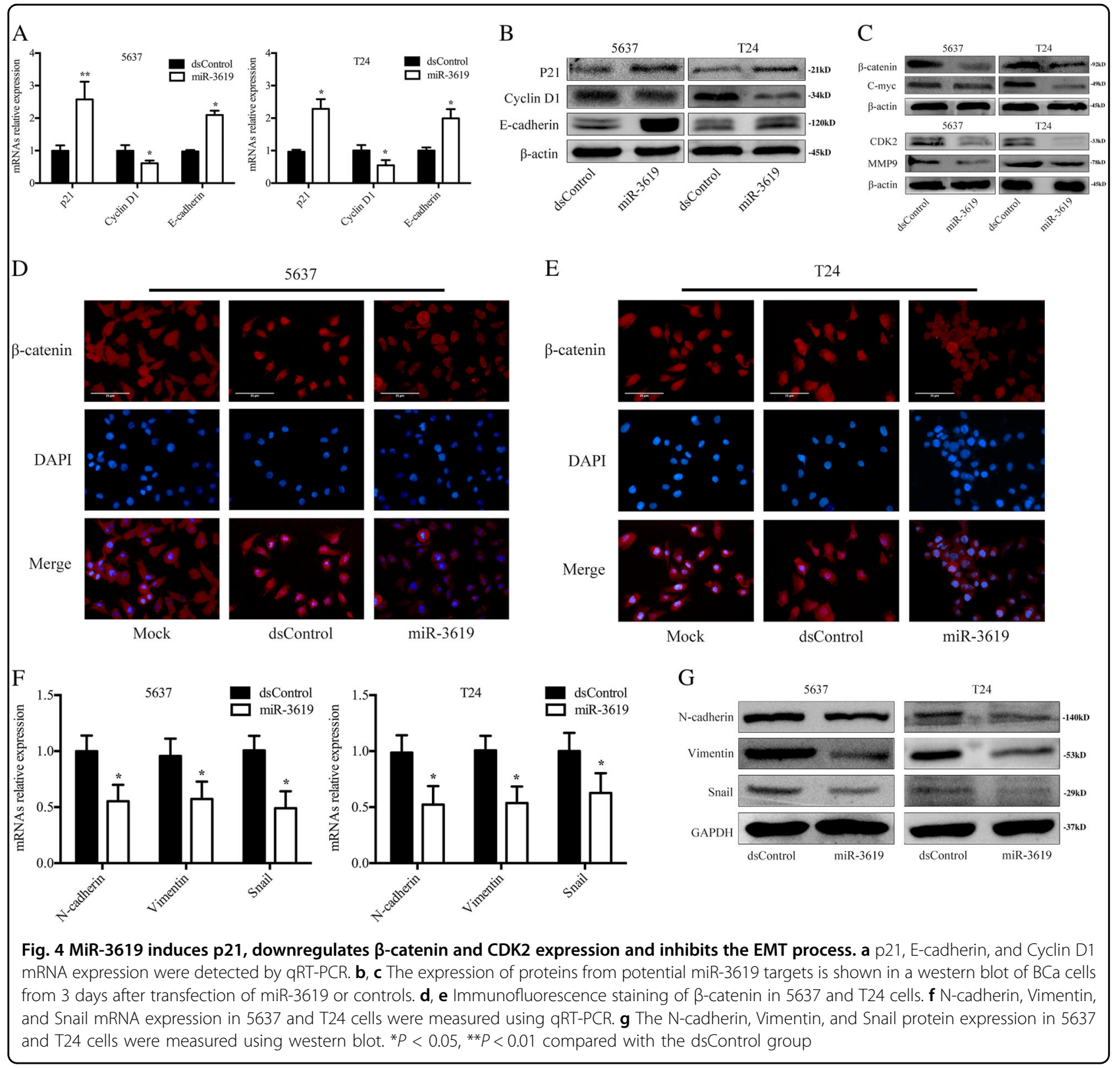

site in its promoter. Seventy-two hours after transfection with biotinylated miR-3619 or the dsControl, the target promoter DNA was pulled down using a corresponding biotin antibody and amplified by qRT-PCR (Fig. 5a). The primer set amplifying the $\mathrm{p} 21$ promoter from $-1245 \mathrm{bp}$ to -1050 bp relative to the TSS (transcription start site) served as a negative control. As seen in Fig. 5b, c, both the $5^{\prime}$-end and 3'-end of the biotin-labeled miR-3619 pulled down promoter proximal DNA (from -275 to -108 ) more obviously than the dsControl in 5637 and T24 cells. In contrast, there was no remarkable change in the covalent link of the miR-3619 and the dsControl RNAs to the negative control region $(-1245$ to -1050$)$. These results indicated that miR-3619 activated p21 gene expression by directly combining with the specific promoter sequence.

Bioinformatics predictions showed that the 3'-UTRs for the human $\beta$-catenin and CDK2 mRNAs contain a potential miR-3619 binding site (Fig. $5 \mathrm{~d}$ ). However, direct evidence for the relationship between miR-3619 and $\beta$ catenin or CDK2 mRNA by luciferase assay was still required. As shown in Fig. 5e, f, the relative reporter gene luciferase activity was significantly weakened when 5637 and T24 cells were cotransfected with luciferase reporter plasmids containing the predicted $\beta$-catenin or CDK2 mRNA target regions and miR-3619 mimics, while the suppression disappeared when the 3'-UTR binding sites were mutated. Moreover, the cotransfection of miR-3619 


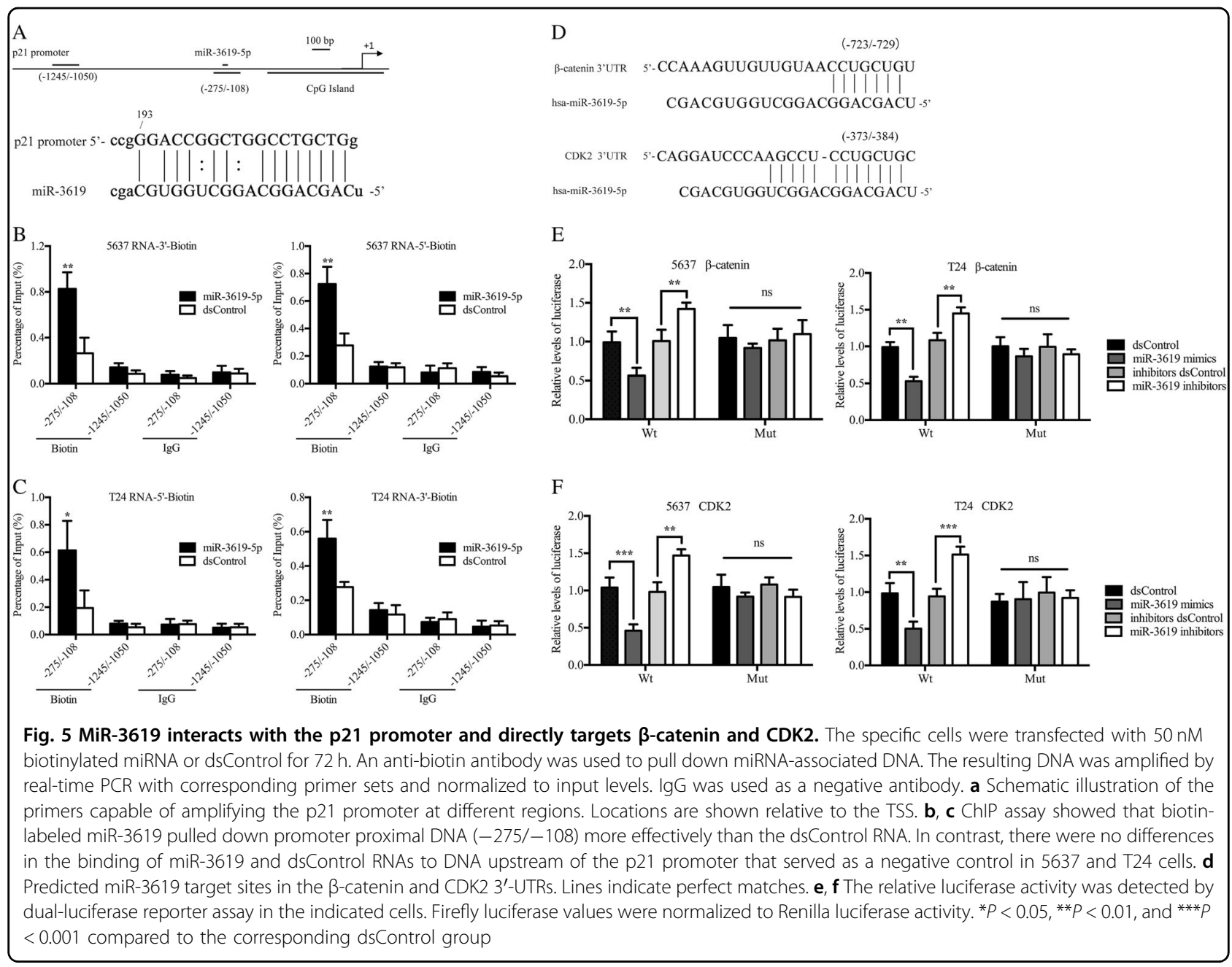

inhibitors with the wild $\beta$-catenin or CDK2 $3^{\prime}$-UTR plasmid led to a relative increase in luciferase signal. These data confirmed that $\beta$-catenin and CDK2 were direct targets of miR-3619.

\section{$\beta$-catenin and CDK2 knockdown mimic the miR-3619- induced phenotype}

There may be a link between the phenotypic changes induced by miR-3619 in BCa cells and the suppression of $\beta$-catenin and CDK2. We introduced short interfering RNAs (siRNAs) targeting $\beta$-catenin or CDK2 into $\mathrm{BCa}$ cell lines. $\beta$-catenin or CDK2 knockdown alone or in combination suppressed 5637 and T24 cell growth, reduced the number of colonies and inhibited 5637 and T24 cell proliferation (Supplementary Figures 3A-F). Additionally, $\beta$-catenin and/or CDK2 knockout increased both early and late stage apoptosis in 5637 and T24 cells (Supplementary Figure 3G). These results are similar to those of miR-3619 overexpression in 5637 and T24 cells.

We subsequently detected the migration and invasion capacities of BCa cells after transfection with si- $\beta$-catenin and/or si-CDK2. As shown in Supplementary Figure 4A$C$, transfection of $\beta$-catenin and/or CDK2 siRNAs led to retarded wound closing and obviously weakened their migration and invasion abilities compared with the dsControl group cells. Besides, $\beta$-catenin knockdown caused a significant decrease in C-myc (Supplementary Figure 4D); CDK2 silencing downregulated MMP9 expression in 5637 and T24 cells. Most importantly, p21 and $\mathrm{E}$-cadherin were upregulated after $\beta$-catenin or CDK2 knockdown in the two cell lines (Supplementary Figure $4 \mathrm{E})$. Together, these data suggested that $\beta$-catenin and CDK2 inhibition was involved in miR-3619-mediated growth arrest in $\mathrm{BCa}$ cells. $\beta$-catenin and CDK2 suppression also had a role in miR-3619-triggered migration and invasion in 5637 and T24 cells.

\section{MiR-3619-induced growth arrest and metastasis inhibition are p21-dependent in BCa cells}

To determine whether there was a relationship between miR-3619-induced inhibition of cell proliferation, migration, and invasion and the expression of $\mathrm{p} 21$ in $\mathrm{BCa}$ cells, 


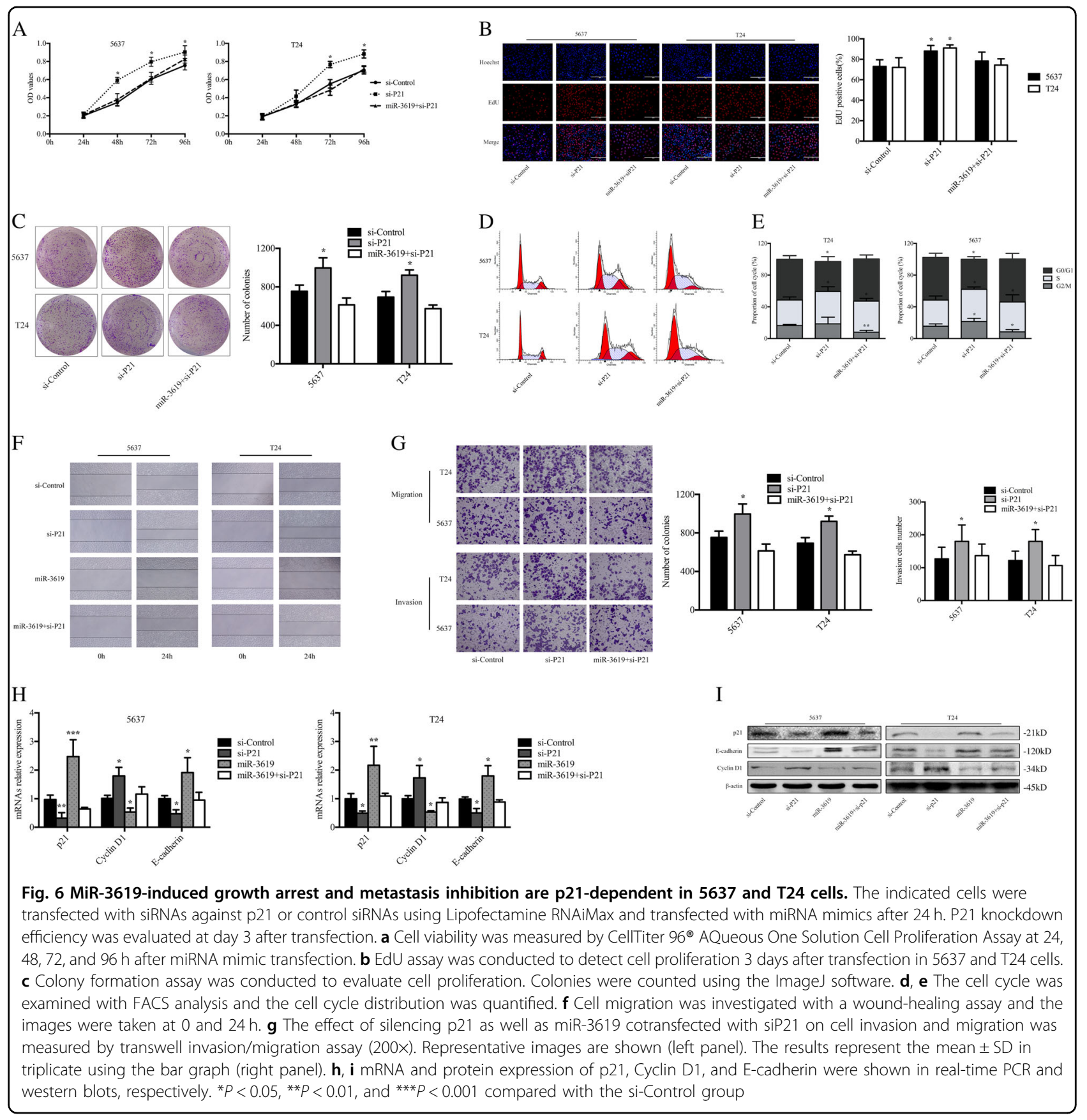

we transfected 5637 and T24 cells with siRNA targeting p21 and miR-3619 mimics, then detected cell proliferation and metastasis. CellTiter $96^{\circledR}$ AQueous One Solution Cell Proliferation assay and EdU assay was conducted and the outcomes showed that overexpression of miR-3619 failed to inhibit the cell proliferation in p21 knockdown 5637 and T24 cells (Fig. 6a, b). Furthermore, we found colony formation ability of the $\mathrm{BCa}$ cells was also restored after cotreatment of si-P21 (Fig. 6c). As shown in Fig. 6d, expression of miR-3619 failed to induce G1/G0 arrest in p21 knockdown 5637 and T24 cells. However, the cells in $\mathrm{S}$ phase were increased and the G2/M phase population was decreased in miR-3619 + si-P21 group compared with si-Control group in BCa cells (Fig. 6e). Wound healing assay was conducted and found miR-3619 also failed to inhibit closing the wound within $24 \mathrm{~h}$ after knockdown p21expression (Fig. 6f). Furthermore, the transwell assay was performed to further assess cells migration and invasion capacity after knockout $\mathrm{p} 21$ genes. As shown in Fig. 6g, depletion of p21 dramatically 
restored cells migration and invasion in response to miR3619 transfection. Consistently, as shown in Fig. 6h, i miR-3619 failed to downregulate Cyclin D1 or upregulate E-cadherin after si-p21 cotransfection in 5637 and T24 cells.

\section{Downregulation of endogenic miR-3619 promotes the growth and metastasis in BCa cells}

We knocked down endogenous miR-3619 in BCa cells to further validate miR-3619 regulation of $\mathrm{p} 21, \beta$-catenin, and CDK2. As shown in Supplementary Figure 5A, p21and E-cadherin mRNAs were downregulated and Cyclin D1 mRNA expression was increased after transfection of miR-3619 inhibitor. Moreover, the p21 protein of anti-miR-3619 group was decreased while $\beta$-catenin and CDK2 were increased compared with anti-Control group (Supplementary Figure 5B). The immunofluorescence demonstrated that the $\beta$-catenin in nucleus was increased after transfection of miR-3619 inhibitor (Supplementary Figure 5C). Besides, growth upregulation was observed upon anti-miR-3619 transfection in cell proliferation assay compared with anti-Control group (Supplementary Figure 5D). Moreover, anti-miR-3619 transfection of 5637 and T24 cells produced a trend towards increased colony formation (Supplementary Figure $5 \mathrm{E}$ and $5 \mathrm{~F}$ ). Furthermore, inhibiting miR-3619 led to decrease in cell apoptosis (Supplementary Figure 5G). The result of transwell assay displaying downregulation of miR-3619 remarkably promoted BCa cells capacity of migration and invasion (Supplementary Figure $5 \mathrm{H}$ ).

\section{Discussion}

Bladder cancer is currently one of the fastest growing tumor in the world. In the past decade, research on metastasis and invasion has been greatly accelerated, which has led to hallmark-targeting therapies development $^{24}$. However, a targeted therapeutic agent may incompletely shut off one or some symbolic abilities by suppressing one crucial pathway in a tumor, allowing partial cancer cells to survive with residual functions until they or their progeny finally accommodate to the selective pressure imposed by the therapy ${ }^{24,25}$. Thus, identifying more important regulators or biomarkers associated with cancer cell proliferation and metastasis and targeting all or most of these supporting pathways therapeutically has become increasingly essential. A few biomarkers have been found to monitor BCa including p21 gene ${ }^{26}$. In this study, we found that $\mathrm{p} 21$ gene also plays an important role in miR-3619 inhibition of BCa. Silencing of endogenous p21 gene in tumor cells significantly reduced the effect of miR-3619 in inhibiting bladder tumors.

MiRNAs are known global regulators of gene expression, and dysregulation of miRNA expression and its associated signaling networks has been implicated in tumor progression ${ }^{27-30}$. In this study, we provided evidence that miR-3619 was a candidate $\mathrm{BCa}$ inhibitor and was associated with directing the $\mathrm{BCa}$ cell phenotype. $\mathrm{BCa}$ cell proliferation in vitro and in vivo and metastasis in vitro were dramatically inhibited by miR-3619 through interactions with p21 promoter-specific sequences. We presented data showing that $\beta$-catenin and CDK2 were direct targets of miR-3619. Wnt/ $\beta$-catenin pathway plays a key role in various biological processes, including cell growth, metastasis, and stem cell self-renewal ${ }^{20,31}$. Dysregulation of the $\mathrm{Wnt} / \beta$-catenin pathway is related to the development of a variety of tumors ${ }^{32}$. We identified that miR-3619 prevented the accumulation of $\beta$-catenin in the cytoplasm or its translocation into the nucleus. Moreover, the Wnt target gene c-Myc was also inhibited after miR3619 overexpression in 5637 and T24 cells, which indicated that miR-3619 inhibited Wnt/ $\beta$-catenin pathway in $\mathrm{BCa}$ cells. EMT process is a crucial initiator and contributor to tumor metastasis. We identified that miR-3619 overexpression enhanced the expression of the epithelial biomarker E-cadherin and suppressed the expression of the mesenchymal biomarkers Vimentin, Snail, and Ncadherin in BCa cells which indicated that miR-3619 suppressed the EMT progression in BCa cells.

The phenomenon of RNA activation (RNAa) was discovered in recent years. Our previous studies have identified that several miRNAs (miR-1236, miR-370, and miR1180) could activate the expression of the tumor suppressor gene $\mathrm{p} 21^{16,33,34}$. We found here that endogenous miR-3619 was downregulated in BCa tissues and cells compared with corresponding normal controls. Moreover, miR-3619 overexpression readily upregulated p21 gene expression by targeting a putative site in its promoter. Experimentally enforced expression of miR-3619 in $\mathrm{BCa}$ cells resulted in cell cycle arrest, senescence acceleration, increased apoptosis, and metastasis suppression. The downregulation of apoptosis is a key factor in tumorigenesis, cancer progression, and chemotherapy resistance in most cancers types ${ }^{35}$. Thus, miR-3619 fulfilled the criteria of a tumor suppressor gene in the context of BCa.

We provided evidence for p21 involvement in miR3619-driven cell cycle arrest, senescence, apoptosis, migration, and invasion. Furthermore, miR-3619 also caused a marked change in the p21 downstream genes, Cyclin D1 and E-cadherin in both T24 and 5637 cells. Furthermore, we found that $\beta$-catenin and CDK2 met the miR-3619 target criteria to create this phenotype. First, they were both downregulated upon miR-3619 transfection into BCa cells. Second, predicted miR-3619 binding sites in their $3^{\prime}$-UTRs reduced the expression of a reporter construct in the presence of miR-3619.

$\beta$-catenin and CDK2 may be linked to negative regulation of the p21 pathway activity. In this study, we found a 
prominent phenotypic similarity between enforced miR3619 expression and $\beta$-catenin/CDK2 knockdown in two $\mathrm{BC}$ a cell lines. We also identified that ectopic expression of $\beta$-catenin/CDK2 statistically increased cell proliferation while inhibiting apoptosis in $\mathrm{BCa}$ cells. This study demonstrated that $\beta$-catenin induced tumorigenicity in $\mathrm{BCa}$ cells by activating the Wnt/ $\beta$-catenin pathway. Furthermore, CDK2 itself also played a crucial role in regulating the proliferation and metastasis of $\mathrm{BCa}$ cells. Additionally, the data indicated that $\beta$-catenin and CDK2 were upregulated in $\mathrm{BCa}$ tissue and several $\mathrm{BCa}$ cell lines compared with the dsControl group. Thus, we confirmed that $\beta$-catenin and CDK2 as direct target genes of miR$3619, \beta$-catenin and CDK2 played vital roles in BCa cell growth and metastasis. In our experiments, $\beta$-catenin and CDK2 expression were significantly downregulated after miR-3619 overexpression in T24 and 5637 cells. Consistently, endogenous miR-3619 knockdown elevated $\beta$ catenin and CDK2 levels and reduced p21 levels, as well as changed their downstream targets expression, respectively. Furthermore, p21 knockdown reverted to the changes in the phenotype transcriptome and cellular proteins induced by miR-3619. Together, these data indicated that $\beta$-catenin and CDK2 inhibition promoted miR-3619-mediated growth inhibition in 5637 and T24 cells, at least in part through the induction of p21.

In summary, our study found that miR-3619 was decreased in BCa tissues and cell lines and that miR-3619 overexpression inhibited cell growth, invasion, and metastasis. Functional experiments also demonstrated that miR-3619 knockdown promoted BCa cell growth and metastasis. Furthermore, p21, $\beta$-catenin, and CDK2 were identified to be direct downstream targets of miR-3619. Taken together, these findings will help us to better understand the mechanisms of miR-3619 in regulating $\mathrm{BCa}$, as well as its role as a potential therapeutic target for the clinical treatment of $\mathrm{BCa}$.

\section{Materials and methods}

\section{Clinical specimens}

The fresh tumor specimens and corresponding paratumor tissues were collected from 33 patients at Tongji Hospital, Tongji Medical College, Huazhong University of Science and Technology (Wuhan, China) between 2014 and 2016 after informed consent and Ethics Committee's approval. All the diagnoses were based on pathology reports.

\section{Cell culture and transfection}

The human BCa cell lines J82, 5637, EJ, and T24 (ATCC, USA) were maintained in RPMI 1640 medium (HyClone, USA) with $10 \%$ fetal bovine serum (Gibco, USA). Both miRNAs and siRNAs were transfected at a final concentration of $50 \mathrm{nM}$ using Lipofectamine
RNAiMax (Invitrogen, USA). Lenti-miR-3619 was used to overexpress miR-3619 following the infection of T24 cells. Lenti-dsControl served as a negative control.

\section{MiRNA and recombinant lentivirus}

The miR-3619-5p mimic, 5' - or 3' - biotin covalently linked miR-3619 mimic, miR-3619-5p inhibitor, miR3619-5p mutation as well as interfering RNAs (si-p21, si$\beta$-catenin, si-CDK2 and si-Control) were chemically synthesized by RiboBio Co., Ltd. (Guangzhou, China). Lenti-dsControl and Lenti-miR-3619 were synthesized by GenePharma (Shanghai, China). The sequences of the dsRNA and miRNA were listed in Supplementary Table 1.

\section{RNA isolation, quantitative real-time PCR, ordinary PCR, and miRNA analysis}

Total RNA was extracted with the TRIzol reagent (Invitrogen, USA). Five hundred nanograms of RNA were reverse transcribed into cDNA. The quantitative real-time PCR was performed on an Mx3000P instrument (Stratagene, USA) with SYBR Premix Ex Taq II (Takara, China). The primers were provided by Invitrogen (Shanghai, China) and were listed in Supplementary Table 2. The partial mRNA PCR product was also analyzed on $2 \%$ agarose gels and visualized.

\section{Western blotting analysis}

Total tissues and cellular proteins were extracted and separated by $10 \%$ sodium dodecyl sulfate polyacrylamide gel electrophoresis (SDS-PAGE) and then transferred onto polyvinylidene fluoride membranes (Millipore, USA). The membranes incubated overnight at $4{ }^{\circ} \mathrm{C}$ with primary antibodies. The antibodies against CDK2, Cyclin D1, $\beta$-catenin, C-myc, N-cadherin, Snail were purchased from Affinity (USA), p21 antibody was from Cell Signaling Technology (USA), E-cadherin antibody was from BD Biosciences (USA), antibodies against MMP9, Vimentin, $\beta$-actin and GAPDH were from Boster (China).

\section{Immunohistochemistry (IHC) analysis}

The tissues were fixed in $4 \%$ paraformaldehyde, routinely dehydrated, paraffin-embedded tissue sections $(5 \mu \mathrm{m})$ were deparaffinized and rehydrated. Paraffin sections were incubated with primary antibodies against $\beta$-catenin (Affinity, USA), CDK2 (Affinity, USA), and p21 (Cell Signaling Technology, USA). The sections were stained with hematoxylin as counterstaining.

\section{Chromatin immunoprecipitation assay (ChIP assay)}

Cells transfected with biotin-labeled miRNAs were used for ChIP assay using a ChIP assay kit (Millipore) at $72 \mathrm{~h}$. Collection of $3 \times 10^{6}$ cells were used for a single immunoprecipitation. One percent formaldehyde was used to 
cross-link chromatin for $10 \mathrm{~min}$ at $37^{\circ} \mathrm{C}$. Then, SDS lysis buffer was used to wash and re-suspend the fixed cells. Biotin (Santa Cruz Biotechnology, USA) antibody and normal rabbit IgG (Millipore, USA) (negative control) were used for immunoprecipitation after the chromatin was precleared with protein A agarose/Salmon sperm DNA overnight at $4{ }^{\circ} \mathrm{C}$. Then, the antibody/antigen/DNA complexes were collected and reversed. The columnpurified (Omega Bio-tek, USA) DNA was used as a template for real-time PCR. The sequences of primers were listed in Supplementary Table 2.

\section{Immunofluorescence staining}

Transfected BCa cells were fixed with $4 \%$ paraformaldehyde, $0.2 \%$ Triton X-100 was used to permeabilize cells, 5\% BSA was used to block the cells. The BCa cells were incubated with primary antibody overnight at $4{ }^{\circ} \mathrm{C}$. Then, the cells were incubated with the secondary antibodies for $1 \mathrm{~h}$ at $37^{\circ} \mathrm{C}$. The coverslips were stained with 4',6-diamidino-2-pheny-lindole (DAPI) after washing three times to visualize the nucleus.

\section{Cell proliferation assay}

CellTiter $96^{\circledR}$ AQueous One Solution Cell Proliferation Assay kit (Promega, USA) was used to assess cell growth as previously described ${ }^{36}$.

\section{Clonogenic survival assay}

The colonies were fixed 10 days later and stained with $0.5 \%$ crystal violet (Sigma, USA) for $30 \mathrm{~min}$ at room temperature. The following formula was used to determine the colony formation rate: Colony formation rate $=$ number of colonies / number of seeded cells $\times 100 \%$.

\section{5-Ethynyl-2'-deoxyuridine proliferation assay}

The Cell-Light EdU DNA cell kit (Ribobio, China) was used to detect cell proliferation. The cells were incubated with $50 \mathrm{mM}$ 5-ethynyl-2'-deoxyuridine (EdU) for $3 \mathrm{~h}$ at $37^{\circ} \mathrm{C}$. The fixed cells were treated with $0.5 \%$ Triton X-100 for $15 \mathrm{~min} ; 1 \mathrm{mg} / \mathrm{ml}$ DAPI (Sigma, USA) was used to stain the cell nuclei for $30 \mathrm{~min}$. The EdU-labeled cells were observed by fluorescence microscopy.

\section{Cell cycle and apoptosis analysis by flow cytometry}

The fixed cells by $70 \%$ cold ethanol were treated with RNase A (Sigma, USA) at $37^{\circ} \mathrm{C}$ for $30 \mathrm{~min}$ and stained with a propidium iodide (PI) (Key-gen Biotech, China) staining solution at $4{ }^{\circ} \mathrm{C}$ for $30 \mathrm{~min}$. Next, the cells were analyzed on an FACS flow cytometer (BD Biosciences, USA). After co-staining the cells with Annexin V-FITC and PI, the cell apoptosis was assessed by flow cytometry according to the manufacturer's instructions (BD Biosciences, USA).

\section{Wound-healing assay}

About $5 \times 10^{5} \mathrm{BCa}$ cells were plated in a six-well plate following transfection. The confluent cell monolayers were scratched. Then, PBS was used to wash the nonadherent cells and serum-free medium was added to the wells. The migrated distances were observed at 0 and $24 \mathrm{~h}$ post-scratch.

\section{Migration and invasion assay}

A 24-well Boyden chamber with an $8 \mu \mathrm{m}$ pore size polycarbonate membrane (Corning, USA) was used to detect the cell motility. For the invasion assay, Matrigel (BD Biosciences, USA) was precoated into the membranes to form a matrix barrier. The membranes were fixed and stained with $0.5 \%$ crystal violet (Sigma, USA).

\section{Subcutaneous xenograft models}

Approximate $5 \times 10^{6}$ infected T24 cells were subcutaneously injected into the right back of 4-week-old male BALB/c-nude mice (Hua Fukang Biological Technology Co., Ltd, China). A caliper was used to measure the tumor length and width every 4 days for 30 days. The xenograft tumor volume was calculated with the following formula: $V=\left(\right.$ length $\times$ width $\left.^{2}\right) / 2$. On day 30 , the tumors were removed and weighed. All the experimental nude mice were cared for and manipulated according to the NIH Animal Care and Use Committee Guidelines of the Experiment Animal Center of Tongji Medical College of Huazhong University of Science and Technology (Wuhan, China).

\section{Statistical analysis}

Data are presented as the mean \pm standard deviation (SD) for three independent experiments. Differences between groups were analyzed by the two-tailed Student's $t$ test using SPSS version 22.0 software (SPSS Inc., Chicago, IL, USA). Statistical significance among three or more groups was based on one-way ANOVA. The correlation between variables was analyzed using Spearman's correlation test. Survival curves were constructed by the Kaplan-Meier method to simultaneously adjust all potential prognostic variables. A $P$ value $<0.05$ was considered to be statistically significant.

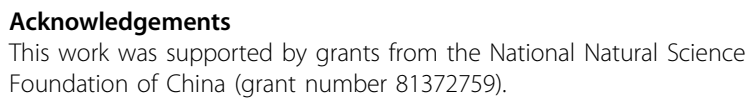

\section{Author details}

${ }^{1}$ Department of Urology, Tongji Hospital, Tongji Medical College, Huazhong University of Science and Technology, No. 1095 JieFang Avenue, 430030 Wuhan, Hubei, China. ${ }^{2}$ Department of Urology, The Affiliated Hospital of Qingdao University, No. 16 Jiangsu Road, Shinan District, 26600 Qingdao, Shandong, China. ${ }^{3}$ Department of Pharmacology, Tongji Medical College, Huazhong University of Science and Technology, No. 1095 JieFang Avenue, 430030 Wuhan, Hubei, China. ${ }^{4}$ Department of Cardiology, Shouguang People's Hospital, 262700 Shouguang, Shandong, China. ${ }^{5}$ Department of Urology, Ruijin 
Hospital, School of Medicine, Shanghai Jiaotong University, 200025 Shanghai, China

\section{Authors' contributions}

Z.C. and Q.Z. were responsible for the experimental design. Q.Z. contributed to the execution of experiments, data statistics, and manuscript composition. S.M. and C.C.L. participated in performing the experiment and in the manuscript mapping and submission. X.H., M.Z., and T.X. participated in the discussion and data interpretation. C.W. and H.X. conceived the study and revised the manuscript. All authors have contributed to and approved the final manuscript.

\section{Conflict of interest}

The authors declare that they have no conflict of interest.

\section{Publisher's note}

Springer Nature remains neutral with regard to jurisdictional claims in published maps and institutional affiliations.

Supplementary Information accompanies this paper at (https://doi.org/ 10.1038/s41419-018-0986-y).

Received: 27 April 2018 Revised: 25 July 2018 Accepted: 26 July 2018 Published online: 20 September 2018

\section{References}

1. Afshari, M., Janbabaei, G., Bahrami, M. A. \& Moosazadeh, M. Opium and bladder cancer: a systematic review and meta-analysis of the odds ratios for opium use and the risk of bladder cancer. PLOS ONE 12, e0178527 (2017).

2. Torre, L. A. et al. Global cancer statistics, 2012. CA Cancer J. Clin. 65, 87-108 (2015).

3. Hirata, $H$. et al. MicroRNA-1826 targets VEGFC, beta-catenin (CTNNB1) and MEK1 (MAP2K1) in human bladder cancer. Carcinogenesis 33, 41-48 (2012).

4. Chamie, K. et al. Recurrence of high-risk bladder cancer: a population-based analysis. Cancer 119, 3219-3227 (2013).

5. Wu, C. L., Ho, J. Y., Chou, S. C. \& Yu, D. S. MiR-429 reverses epithelialmesenchymal transition by restoring E-cadherin expression in bladder cancer. Oncotarget 7, 26593-26603 (2016).

6. Lewis, B. P., Burge, C. B. \& Bartel, D. P. Conserved seed pairing, often flanked by adenosines, indicates that thousands of human genes are microRNA targets. Cell 120, 15-20 (2005)

7. $\mathrm{Yu}, \mathrm{X}$. et al. MicroRNA-10b promotes nucleus pulposus cell proliferation through RhoC-Akt pathway by targeting HOXD10 in intervetebral disc degeneration. PLOS ONE 8, e83080 (2013).

8. Yeung, M. L. \& Jeang, K. T. MicroRNAs and cancer therapeutics. Pharm. Res. 28, 3043-3049 (2011)

9. Vinall, R. L., Ripoll, A. Z., Wang, S., Pan, C. X. \& deVere White, R. W. MiR-34a chemosensitizes bladder cancer cells to cisplatin treatment regardless of p53Rb pathway status. Int. J. Cancer 130, 2526-2538 (2012).

10. Kurashige, J. et al. MicroRNA-200b regulates cell proliferation, invasion, and migration by directly targeting ZEB2 in gastric carcinoma. Ann. Surg. Oncol. 19 (Suppl 3), S656-S664 (2012).

11. Zhang, X. et al. MicroRNA-7 arrests cell cycle in G1 phase by directly targeting CCNE1 in human hepatocellular carcinoma cells. Biochem. Biophys. Res. Commun. 443, 1078-1084 (2014).
12. Huber et al. Deltex-1 activates mitotic signaling and proliferation and increases the clonogenic and invasive potential of U373 and LN18 glioblastoma cells and correlates with patient survival. PLOS ONE 8, e57793 (2013).

13. Pierzynski, J. A. et al. Genetic variants in the Wnt/beta-catenin signaling pathway as indicators of bladder cancer risk. J. Urol. 194, 1771-1776 (2015).

14. McConkey, D. J. et al. Role of epithelial-to-mesenchymal transition (EMT) in drug sensitivity and metastasis in bladder cancer. Cancer Metastas-. Rev. 28, 335-344 (2009)

15. Gavert, N. \& Ben-Ze'ev, A. Epithelial-mesenchymal transition and the invasive potential of tumors. Trends Mol. Med. 14, 199-209 (2008).

16. Wang, C. et al. Up-regulation ofp21(WAF1/CIP1) by miRNAs and its implications in bladder cancer cells. FEBS Lett. 588, 4654-4664 (2014).

17. Huang, Q. et al. The microRNAs miR-373 and miR-520c promote tumour invasion and metastasis. Nat. Cell Biol. 10, 202-210 (2008).

18. Betel, D., Wilson, M., Gabow, A., Marks, D. S. \& Sander, C. The microRNA.org resource: targets and expression. Nucleic Acids Res. 36(Database issue), D149-D153 (2008).

19. John, B. et al. Human microRNA targets. PLoS Biol. 2, e363 (2004).

20. Kim, W., Kim, M. \& Jho, E. H. Wnt/beta-catenin signalling: from plasma membrane to nucleus. Biochem. J. 450, 9-21 (2013).

21. Huang, V. et al. Upregulation of Cyclin B1 by miRNA and its implications in cancer. Nucleic Acids Res. 40, 1695-1707 (2012).

22. $\mathrm{Hu}, \mathrm{J}$. et al. Promoter-associated small double-stranded RNA interacts with heterogeneous nuclear ribonucleoprotein $A 2 / B 1$ to induce transcriptional activation. Biochem. J. 447, 407-416 (2012).

23. Janowski, B. A. et al. Activating gene expression in mammalian cells with promoter-targeted duplex RNAs. Nat. Chem. Biol. 3, 166-173 (2007).

24. Hainaut, P. \& Plymoth, A. Targeting the hallmarks of cancer: towards a rational approach to next-generation cancer therapy. Curr. Opin. Oncol. 25, 50-51 (2013).

25. Hanahan, D. \& Weinberg, R. A. Hallmarks of cancer: the next generation. Cell 144, 646-674 (2011).

26. Rosenblatt, R. et al. Current status of prognostic immunohistochemical markers for urothelial bladder cancer. Tumour Biol. 29, 311-322 (2008).

27. Calin, G. A. \& Croce, C. M. MicroRNA signatures in human cancers. Nat. Rev. Cancer 6, 857-866 (2006).

28. Di Leva, G., Garofalo, M. \& Croce, C. M. MicroRNAs in cancer. Annu. Rev. Pathol. 9, 287-314 (2014)

29. Kasinski, A. L. \& Slack, F. J. Epigenetics and genetics. MicroRNAs en route to the clinic: progress in validating and targeting microRNAs for cancer therapy. Nat. Rev. Cancer 11, 849-864 (2011).

30. Perl, A. K., Wilgenbus, P., Dahl, U., Semb, H. \& Christofori, G. A causal role for Ecadherin in the transition from adenoma to carcinoma. Nature 392, 190-193 (1998).

31. Gao, C. \& Chen, Y. G. Dishevelled: the hub of Wnt signaling. Cell. Signal. 22 717-727 (2010)

32. Fodde, R. \& Brabletz, T. Wnt/beta-catenin signaling in cancer stemness and malignant behavior. Curr. Opin. Cell Biol. 19, 150-158 (2007).

33. Wang, $C$. et al. Promoter-associated endogenous and exogenous small RNAs suppress human bladder cancer cell metastasis by activatingp21 (CIP1/NAF1) expression. Tumour Biol. 37, 6589-6598 (2016).

34. Ge, Q. et al. The suppressive effects of miR-1180-5p on the proliferation and tumorigenicity of bladder cancer cells. Histol. Histopathol. 32, 77-86 (2017)

35. Strasser, A., Cory, S. \& Adams, J. M. Deciphering the rules of programmed cell death to improve therapy of cancer and other diseases. EMBO J. 30 3667-3683 (2011)

36. Wang, $C$. et al. A new double stranded RNA suppresses bladder cancer development by upregulating p21 (Waf1/CIP1) expression. Biomed. Res. Int 2015, 304753 (2015). 